\title{
Atomic-scale study of dry sliding friction
}

\author{
A. Buldum and S. Ciraci \\ Department of Physics, Bilkent University, Bilkent 06533, Ankara, Turkey \\ (Received 17 June 1996; revised manuscript received 25 September 1996)
}

\begin{abstract}
We present a theoretical study of dry sliding friction, which has a close bearing on the experiments done by using the atomic and friction force microscope. By performing atomic-scale calculations for the friction between a single atom and monoatomic infinite chain, we examined the effect of various material parameters on the stick-slip motion. We found that the perpendicular elastic deformation of the substrate that is induced by the sliding object is crucial for the energy damping in friction. In this case, the average friction force strongly depends on the perpendicular force constant of the substrate and the friction constant varies with the normal force. In particular, soft materials that continue to be elastic for a wide range of perpendicular compression may exhibit a second state. As a result, the hysteresis curve in the stick-slip motion becomes anisotropic.
\end{abstract} [S0163-1829(97)04804-2]

\section{INTRODUCTION}

The relative motion of two objects at close proximity (sliding, rolling, or motion in the perpendicular direction) induces nonconservative forces that resist the motion. This phenomenon is called friction and is relevant for various disciplines in science and technology. ${ }^{1-4}$ The origin of the friction force, and the energy damping therefrom, is the shortand long-range interactions between two objects. Depending on the distance between objects and also on their relative lateral positions, the magnitude of the interaction potential varies and it can be either attractive or repulsive. The invention of the atomic force microscope ${ }^{5}$ (AFM) and the frictional force microscope ${ }^{6,7}$ (FFM) has made an important impact on the science of friction or tribology. Nowadays, perpendicular and lateral atomic forces in the range of nanonewtons (which is $10^{-9} \mathrm{~N}$ and $0.62415 \mathrm{eV} / \AA$ ) can be measured with precision by using these microscopes. ${ }^{8-10}$

The moving objects either are in direct contact through the asperities or lubricants are introduced between them to reduce friction. In boundary lubrication, foreign atoms with monolayer coverage prevent the surfaces of moving objects from making adhesive contact. The dry sliding friction between atomically flat, commensurate or incommensurate sliding surfaces perhaps is the simplest but most fundamental type of friction in tribology. Depending on the conditions, it may include several interesting phenomena such as adhesion, wetting and atom transfer, strain-induced phase transition and local surface reconstruction, anisotropy in stick-slip motion, and dissipation of a local, nonequilibrium distribution of phonons. Whatever the type and the scale of the friction, the atomic process between the sliding or moving objects is crucial for friction. ${ }^{11}$ During various atomic processes the energy of motion is damped by phononic and electronic mechanisms. The nonequilibrium phonon density created locally in the course of sliding is dissipated by phonon-phonon and electron-phonon coupling. The energy dissipation may involve also electron-hole creation and charge-density waves. $^{12}$

Experimental investigations of the interaction between solid surfaces in dry friction and between surface and lubri- cant atoms in boundary lubrication have shed light on the underlying microscopic mechanism of friction. In the meantime, theoretical studies using atomic models ${ }^{13-18}$ and largescale molecular-dynamics simulations ${ }^{19-21}$ have provided insight for a better understanding. Nowadays, researchers have come up with interesting results such as a superlubricant state at high sliding velocities ${ }^{22}$ and the possibility of the nearly frictionless sliding in mesoscopic solids. ${ }^{23}$

The atomic-scale analysis of the interaction between sliding surfaces is necessary to understand the nonconservative lateral forces and the mechanism of energy dissipation in friction. It is hoped that with the knowledge gained this way one will able to provide means towards a better control of friction and hence reduce the loss of energy. With this motivation our study presents an analysis of the interaction in dry sliding friction. First, the nature of the interactions and lateral and perpendicular forces generated therefrom are discussed. The effect of the lateral and perpendicular deformations that are induced by a sliding object (represented by a single atom) on an infinite atomic chain is investigated. The present model is comprised of features that were not included in the models used in the earlier theoretical studies. The results obtained from this work indicate that the elasticity of the surface in the perpendicular direction has a significant effect on the friction and may give rise to a second state for a certain range of elastic constants and normal force.

\section{NATURE OF INTERACTIONS BETWEEN SLIDING SURFACES}

Short-range and long-range interactions occur between the surfaces of sliding objects. The short-range interaction is comprised of the Coulomb repulsion of ions and the attractive electron-ion interaction. ${ }^{2}$ In spite of the fact that repulsive interaction is long ranged, it is screened by the electronic charge density that decays exponentially above the surface. The interaction energy $E_{i}(\vec{r})$ between two atomically flat and commensurate metal slabs $(A$ and $B)$ can be obtained by comparing the total energies ${ }^{24}$ of the individual slabs with the total energy of two interacting slabs $(A+B)$. The relative position of the slabs is given by the vector 
$\vec{r}=\vec{r}(x, y, z)$ between two points $O_{A}$ and $O_{B}$ fixed in the slabs $A$ and $B$, respectively. It is assumed that these points are far from the interaction region, so they are not affected by the induced deformations in $A$ and $B$. For a given separation $z=z_{0}, E_{i}\left(x, y, z_{0}\right)$ has a two-dimensional corrugation. The minimum of the interaction energy and the corresponding height $z=z_{e}(x, y)$ for a given lateral position $(x, y)$ is determined from $\left[\partial E_{i}(\vec{r}) / \partial z\right]_{x, y}=0$. The lowest value of $E_{i}$ (or highest adhesion energy) generally occurs when an atom of one commensurate surface faces the hollow $(H)$ site at the other surface. When $\left|z-z_{e}\right|>0, E_{i}$ increases and a normal attractive (repulsive) force acts on the slabs if $z>z_{e}$ $\left(z<z_{e}\right)$. An external normal force $F_{N}$ applied to the object sets the separation $\bar{z}\left(x, y ; F_{N}\right)$ at a given lateral position $(x, y)$. If one of the commensurate slabs $B$ slides above $A$ under the constant normal force $F_{N}, z=\bar{z}\left(x, y ; F_{N}\right)$ and $E_{i}(x, y, \bar{z})$ varies with the lateral position. The corrugation of $E_{i}$ under the constant normal force is the prime cause of friction since it leads to the lateral force

$$
\vec{F}_{L}=-\left\{\left[\frac{\partial E_{i}}{\partial x}\right]_{z=\bar{z}} \vec{i}+\left[\frac{\partial E_{i}}{\partial y}\right]_{z=\bar{z}} \vec{j}\right\} .
$$

By definition this force is conservative, but part of it becomes nonconservative owing to the stick-slip motion. During the slip, the part of the energy that is stored during sticking is damped by creating a local, nonequilibrium phonon density. The sliding object normally slips less then a lattice parameter, but at certain conditions it can slip more than a unit cell. The van der Waals interaction between the atoms of the two slabs $A$ and $B$ is weak. However, owing to its longer range, the forces on atoms farther away can be approximated by an integral over body forces. This gives a relatively strong but essentially uncorrugated attraction. Consequently, the long-range interaction does not contribute directly to the corrugation of $F_{L}$, except that it affects the magnitude of the applied normal force. The above arguments are given for a general case in two-dimensions, but they can apply also to the one-dimensional friction.

\section{MODEL CALCULATIONS}

Earlier, various features of friction with various lubricant molecules and atoms have been explored by using classical molecular-dynamics method. ${ }^{19-21}$ Even simple models such as Tomlinson's ${ }^{13}$ and Frenkel and Kontorova's ${ }^{14}$ methods have been useful to study dry sliding friction. The model used in the present study aims to explore the effects of various material parameters on the friction. In particular, the friction and stick-slip motion are analyzed in the presence of local elastic deformation in the perpendicular direction. In this respect, our model described in Fig. 1 is different from earlier models.

The sliding object is represented by a single atom, which is sensitive to the atomic structure and the energy corrugation of the substrate. It has coordinates $\vec{\rho}_{t}\left(x_{t}, z_{t}\right)$. A sliding object incorporating several atoms could have contributed to energy damping. This would be only a further elaboration of the present model. The agent that pushes the sliding object has coordinates $\vec{\rho}_{D}\left(x_{D}, z_{D}\right)$, where $z_{D}=z_{t}$. Note that the

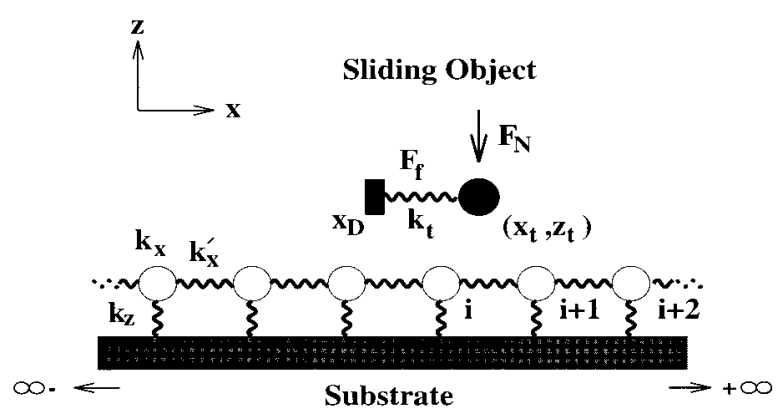

FIG. 1. Atomic model used to study the friction force $F_{f}$ and the stick-slip motion. $F_{N}$ is the normal loading force, $\left(x_{t}, z_{t}\right)$ are the coordinates of the moving agent represented by a single atom, $x_{D}$ is the position of the moving agent, and $k_{t}, k_{x}, k_{x}^{\prime}$, and $k_{z}$ are the spring constants described in the text.

motion of atoms occurs in the lateral $(x)$ and perpendicular $(z)$ directions. The lateral force, acting on the sliding atom is given by $F_{L}=k_{t}\left(x_{t}-x_{D}\right)$ (assuming that $F_{L}=0$ for $x_{D}=x_{t}$ ), which corresponds to the friction force. The interaction potential between the sliding atom and substrate atoms $V_{t i}\left(\left|\vec{\rho}_{t}-\vec{\rho}_{i}\right|\right)$ are represented by a Lennard-Jones pair potential having parameters $\epsilon=0.84 \mathrm{eV}$ and $r_{0}=2.56 \AA$. Those parameters are fitted to the physical properties of the $\mathrm{Ni}$ metal. The substrate is modeled by an infinite chain of atoms. This way various edge effects due to the finite size of the substrate are avoided. $\vec{\rho}_{i}\left(x_{i}, z_{i}\right)$ and $\vec{\rho}_{0 i}\left(x_{i 0}, z_{i 0}\right)$ denote the equilibrium positions of the chain atoms with and without an interaction due to the sliding object, respectively. The cohesion of the substrate is provided by the interchain potential. The total potential of the whole system (sliding object and the substrate) is given by

$$
\begin{aligned}
V_{T}= & \sum_{i} V_{t i}\left(\left|\vec{\rho}_{t}-\vec{\rho}_{i}\right|\right)+\frac{1}{2} k_{t}\left(x_{t}-x_{D}\right)^{2}+\sum_{i} \frac{1}{2} k_{x}\left(x_{i}-x_{i, 0}\right)^{2} \\
& +\sum_{i} \frac{1}{2} k_{x}^{\prime}\left[\left(x_{i+1}-x_{i+1, o}\right)-\left(x_{i}-x_{i, 0}\right)\right]^{2}+\sum_{i} V_{p, i} \\
& +F_{N} z_{t} .
\end{aligned}
$$

Here $V_{p, i}$ is the potential of substrate atoms in the perpendicular direction, which is taken to be harmonic, i.e., $V_{p, i}=k_{z}\left(z_{i}-z_{i 0}\right)^{2} / 2$. The total potential $V_{T}$ as expressed in Eq. (2) is reminiscent of the interaction energy $E_{i}$ discussed in Sec. II. However, the electronic contribution to $V_{T}$ is robust. The last term in $V_{T}$ indicates that an external perpendicular force $F_{N}$ is acting on the sliding atom. This corresponds to the normal loading force

$$
F_{N}=-\sum_{i}\left[\frac{\partial V_{t i}}{\partial z_{t}}\right]_{\bar{z}_{t}}
$$

and sets $z_{t}=\overline{z_{t}}$ for a given lateral position $x_{t}$. Then the equation of motion of the moving atom under the constant normal force is

$$
m \ddot{x}_{t}+\sum_{i} \partial V_{t i} /\left.\partial x_{t}\right|_{z=\bar{z}_{t}}+F_{N} \partial \overline{z_{t}} / \partial x_{t}+k_{t}\left(x_{t}-x_{D}\right)=0 .
$$



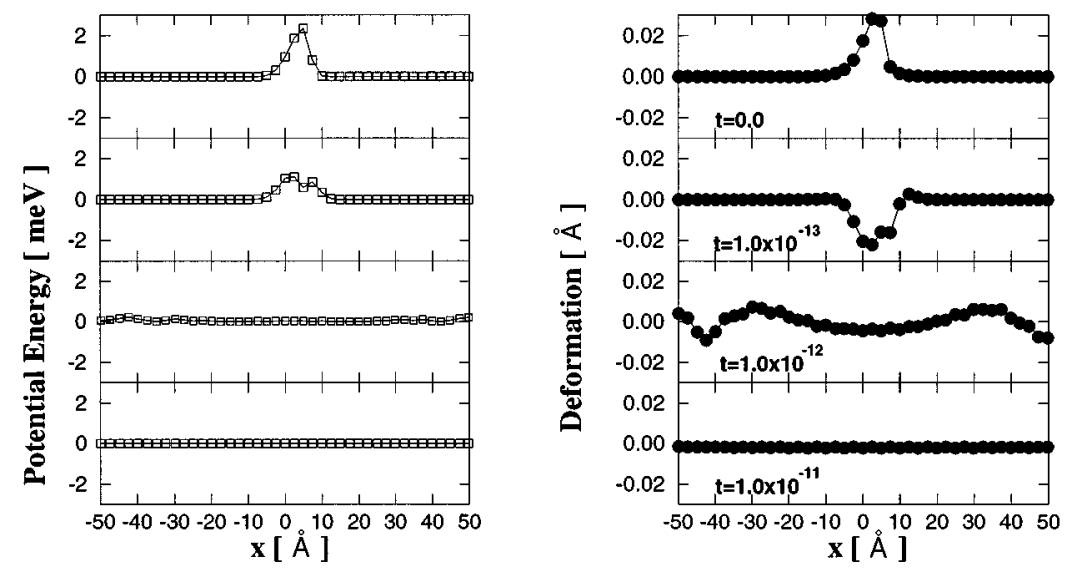

FIG. 2. Propagation of the local potential (strain) energy and deformation created in an infinite 1D substrate. Atoms are allowed to move in the $x$ direction since $k_{z}$ is taken to be infinite.
Similarly, the equation of motion for the substrate atoms

$$
\begin{array}{r}
m \ddot{x}_{i}+\partial V_{t i} / \partial x_{i}+k_{x}\left(x_{i}-x_{i, 0}\right)+2 k_{x}^{\prime} x_{i}-k_{x}^{\prime}\left(x_{i+1}+x_{i-1}\right)=0, \\
i=0, \pm 1, \pm 2, \ldots .
\end{array}
$$

In the present model, the sliding atom (or object) applies a force to the substrate and induces local deformation. The strain energy stored in this local deformation spreads out in the substrate. This is the path of the energy transfer from the moving object to the substrate leading to the dissipation. Figure 2 illustrates the propagation (dissipation) of the local strain energy and deformation induced by the moving object just before the start of the slip. At this moment the moving object is removed and atoms are allowed to move only in the $x$ direction by taking $k_{z}$ to be infinite. This can be viewed as the equilibration of a nonequilibrium phonon distribution induced at the close proximity of the tip. The deformation propagates with an average speed of $\sim 3000 \mathrm{~m} / \mathrm{s}$. As the speed of the moving object approaches this velocity the dynamical solution becomes important. At this point the following comments are in order. Since the present model has only limited extent in the perpendicular direction, the propagation of the strain energy along the $z$ direction is not considered. The longitudinal and the transversal motion of the substrate atoms are coupled through the two-body potential $V_{t i}$. However, the transversal motions of atoms become decoupled if they are induced by a local deformation in the absence of the sliding object. As a result, the propogation of the longitudinal mode is not affected in any essential manner by freezing the transversal motion. While part of the mechanical energy transferred from the sliding object to the substrate quickly dissipates through the infinite chain in the longitudinal mode, the remaining part sets vibrations on the atoms left behind the sliding object. In the actual friction, the local deformation creates a local nonequilibrium phonon distribution in the sample as well as in the sliding object. The local strain propogates with the speed of sound and the local nonequilibrium phonon distribution fades away by electronphonon and mainly phonon-phonon interactions.

In the present study we consider the low sliding speeds in the range of $v_{D}=d x_{D} / d t \sim 4 \AA / \mathrm{s}$. This is relevant for the AFM or the FFM. Furthermore, we assume that the normal force $F_{N}$ does not cause any kind of plastic deformation or wear; hence the energy transferred to the substrate by an induced local deformation spreads with the speed of sound and is dissipated almost suddenly. These conditions justify the quasistatic approximation, in which the friction force $F_{f}$ at a given time $t$ is equal to the lateral force $F_{L}$. This requires the determination of the actual positions of substrate atoms $\left\{x_{i}, z_{i}\right\}$ for a given $x_{D}$ and $F_{N}$. Since all these coordinates are interrelated through Eq. (2), they can be calculated by using tedious iterative procedures. The calculation of the interaction potential $\Sigma_{i} V_{t i}$ involves 141 atoms at close proximity to the sliding object. Only 60 atoms out of 141 are allowed to relax under the interaction potentials. The rest of the substrate atoms that are far from the sliding object are kept in their original position since their displacements under the force exerted by the other atoms are insignificant. For $x_{D}$ at time $t$ we start by determining the equilibrium positions of the atoms for a given $\left(x_{t}, z_{t}\right)$ by using equilibrium conditions for the forces obtained from the interaction potential. Note that the sliding atom is under the forces $F_{N}, F_{L}$, and the force derived from interaction potential $\sum_{i} V_{t i}\left(\left|\vec{\rho}_{t}-\vec{\rho}_{i}\right|\right)$. In the iteration cycles $z_{t}$ is varied continuously to find $\overline{z_{t}}$, so that the normal force becomes equal to the desired value $F_{N}$. The lateral coordinate $x_{t}$ of the sliding object is varied to balance $F_{L}$. Upon reaching the equilibrium condition at $x_{D}$, the friction force $F_{f}$, which is equal to $F_{L}$, is calculated from the actual values of $x_{D}$ and $x_{t}$. By increasing $x_{D}$ by $v_{D} \Delta t$ all steps of iterations are repeated to find $F_{f}$ for this new value of $x_{D}$. This way $F_{f}$ versus $x_{D}$ (or $F_{f}$ versus $t$ ) curves are obtained for right sliding or left sliding moving object. This leads to a hysteresis since the average of $\bar{F}_{L}, \quad \int_{x_{D 1}}^{x_{D 2}} F_{L} d x_{D} /\left(x_{D 2}-x_{D 1}\right)$ is finite and is equal to average friction force $F_{f}$.

\section{RESULTS AND DISCUSSION}

The stick-slip motion, in particular $F_{f}$ and the hysteresis curve in dry sliding friction between atomically flat surfaces, is strongly dependent on material parameters (i.e., harmonic and anharmonic terms of the interaction potential) and $F_{N}$. In the model described by Fig. 1, the force constant $k_{t}$ of the sliding object and those of the substrate $k_{x}, k_{x}^{\prime}, k_{z}$ and the interaction potential $V_{t i}$ between the sliding object and the substrate are of crucial importance. The earlier treatments that assumed a rigid substrate surface did not take into account the deformation induced by the moving object. Using the quasistatic approximation, we calculated the hysteresis 


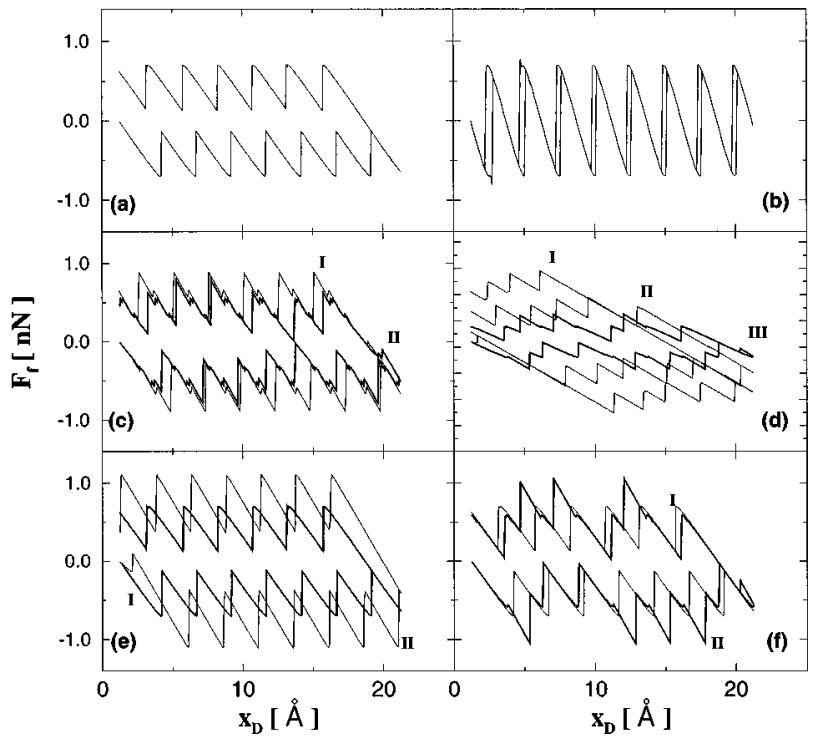

FIG. 3. Hysteresis curves (i.e., variation of $F_{f}$ in a cycle of $x_{D}$ ) calculated for different material parameters and normal loading force. (a) $F_{N}=0 \mathrm{nN} ; k_{t}=0.2, k_{x}=4.3, k_{x}^{\prime}=5.8$, and $k_{z}=10 \mathrm{eV} / \AA$ 2. (b) Same as (a) except $k_{t}=0.8 \mathrm{eV} / \AA^{2}$. (c) $F_{N}=0 ; k_{z}=10$; $k_{t}=0.2 \mathrm{eV} / \AA^{2} ; \mathrm{I}, k_{x}=k_{x}^{\prime}=1.5 \mathrm{eV} / \AA^{2} ; \mathrm{II}, k_{x}=k_{x}^{\prime}=1.7 \mathrm{eV} / \AA^{2}$. (d) $F_{N}=0.2 \mathrm{nN} ; k_{x}=4.3 ; k_{x}^{\prime}=5.8 ; k_{t}=0.2 \mathrm{eV} / \AA^{2} ; \mathrm{I}, k_{z}=2.5 ; \mathrm{II}$, $k_{z}=5 ;$ III, $k_{z}=10 \mathrm{eV} / \AA^{2}$. (e) $k_{t}=0.2 ; k_{x}=4.3 ; k_{x}^{\prime}=5.8 ; k_{z}=10$ $\mathrm{eV} / \AA^{2}$; I, $F_{N}=0 \mathrm{nN}$; II, $F_{N}=1 \mathrm{nN}$. (f) Same as (e) except II, $F_{N}=0.2 \mathrm{nN}$.

curve [i.e., the $F_{f}\left(x_{D}\right)$ curve in a cycle of $x_{D}$ ] and examined the effect of material parameters and $F_{N}$ on the average friction force $\bar{F}_{f}$. Figure 3 summarizes our results.

According to the definition of friction force in the present model, the elasticity of the sliding object $k_{t}$ is essential for the stick-slip motion and $F_{f}$. In fact, the loss of energy in the course of slipping decreases as $k_{t}$ increases, and eventually the bistability leading to the slip does not occur when $k_{t}$ exceeds the value set for a given substrate having the corrugation $F_{L}\left(x ; F_{N}\right)$. In Figs. 3(a) and 3(b) the effect of $k_{t}$ in the energy damping is seen by comparing the area in the hysteresis curves resulting for two different $k_{t}$. For a given $k_{t}$, the friction force $F_{f}$ is strongly dependent on the lateral and perpendicular force constants of the substrate. The energy damping increases with decreasing lateral force constants. For example, by comparing hysteresis curves in Fig. 3(c) one concludes that between two different sets of lateral force constants $k_{x}, k_{x}^{\prime} \simeq 1.5,1.7 \mathrm{eV} / \AA^{2}$ (at fixed loading force $F_{N}=0, k_{t}=0.2, k_{z}=10 \mathrm{eV} / \AA^{2}$ ) the strongest damping of energy occurs for $k_{x}, k_{x}^{\prime}=1.5 \mathrm{eV} / \AA^{2}$. In general, the average energy damping increases with decreasing $k_{z}$ at a fixed $F_{N}$ and also with increasing $F_{N}$. However, $\bar{F}_{f}\left(k_{z}\right)$ at constant $F_{N}$ and $\bar{F}_{f}\left(F_{N}\right)$ at constant $k_{z}$ are not linear and require a detailed analysis. We present this analysis in Figs. 3-5. The essential aspects of nonlinearity, which were not taken into account before, are the deformation-induced modification of the interaction potential and its anharmonicity. In Fig. 3(d) the hysteresis curves corresponding to $k_{z}=10,5$, and $2.5 \mathrm{eV} /$ $\AA^{2}\left(\right.$ at $F_{N}=0.2 \mathrm{nN}, k_{x}=4.3, k_{x}^{\prime}=5.8$, and $\left.k_{t}=0.2 \mathrm{eV} / \AA^{2}\right)$ show that the energy damping (or $\bar{F}_{f}$ ) increases with decreasing $k_{z}$ in the range $2.5<k_{z}<10$. Later in this section we
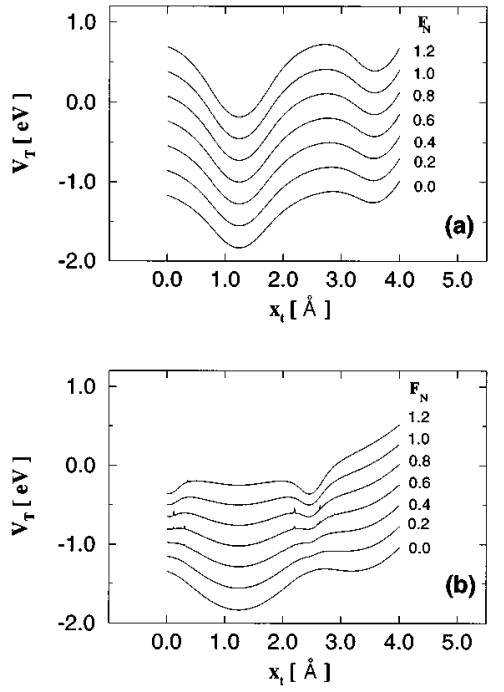

FIG. 4. Total potential $V_{T}$ versus $x_{t}$ calculated for various normal forces $F_{N}$ in nN. (a) Rigid substrate in the perpendicular direction, i.e., $k_{z}=\infty$ and (b) $k_{z}=10 \mathrm{eV} / \AA^{2}$.

will see that the behavior of $\bar{F}_{f}\left(k_{z}\right)$ is not straightforward, however. In Figs. 3(e) and 3(f) the hysteresis curves for $F_{N}=0,0.2$, and $1 \mathrm{nN}$ (with $k_{x}=4.3, k_{x}^{\prime}=5.8$, and $k_{t}=0.2$ $\mathrm{eV} / \AA^{2}$ ) show that the energy damping increases with increasing $F_{N}$. It is also seen that the stick-slip motion becomes irregular for a certain range of $F_{N}$ and in the same hysteresis curve two different state stick-slip motions (or some kind of anisotropy) are distinguished. Such an effect dissapears as $k_{z} \rightarrow \infty$ (i.e., the substrate surface becomes rigid) or the variation of $V_{T}$ in the perpendicular direction becomes strongly anharmonic. Normally, one expects that the profile of $F_{f}\left(x_{D}\right)$ is uniform even if $F_{N} \neq 0$ since the
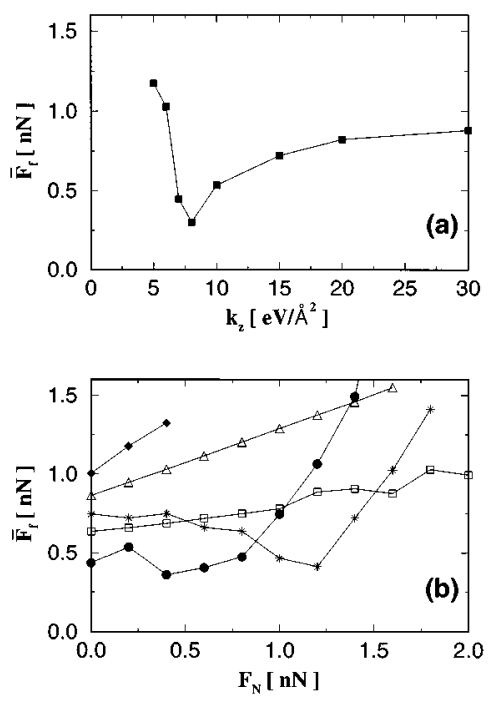

FIG. 5. (a) Variation of the average friction force as a function of force constant in the perpendicular direction $k_{z} \cdot F_{N}=0.2 \mathrm{nN}$. (b) Average friction force versus normal loading force $F_{N}$ calculated for various $k_{z}$. Filled squares, triangles, stars, filled circles, and empty squares correspond to $k_{z}=5, \infty, 15,10 \mathrm{eV} / \AA^{2}$, and anharmonic $V_{p, i}$, respectively. 
elastic deformation is continuous. However, due to the corrugation inversion, a discontinuous change in the elastic deformation can take place at certain range of $F_{N}$. Then the motion makes a transition from one minimum (state 1) to another minimum (state 2). This interesting finding is clarified in Fig. 4(a). As seen, $V_{T}$ is low at the $H$ site (between two substrate atoms), but increases at the $T$ site (on top of the substrate atom). The corrugation $\Delta V_{T}=V_{T}(T)-V_{T}(H)$ increases with increasing normal force for $k_{z} \gg 1$. On the other hand, $\Delta V_{T}$ varies with the normal force if the substrate surface is elastic in the perpendicular direction. In Fig. 4(b) the corrugation $\Delta V_{T}$ is positive for $F_{N}=0$, but decreases with increasing $F_{N}$; eventually the corrugation is inverted at some range of $F_{N}$. The inversion of $\Delta V_{T}$ is related with the large elastic deformation of the substrate in the perpendicular direction. Our results predict that the corrugation inversion occurs for substrates that have small $k_{z}$ for a wide range of perpendicular elastic deformation and are closely related to the second state in the stick-slip motion: For $F_{N}=0, F_{f}$ has a well-defined value prior to the slip and the profile of $F_{f}\left(x_{D}\right)$ is uniform. Our calculations predict that the profile of $F_{f}\left(x_{D}\right)$ exhibits anisotropy at a certain range of $F_{N}$. The range of $F_{N}$ where anisotropy in the stick-slip motion occurs depends on the value of $k_{z}$. Upon the onset of the anisotropy the stick-slip motion makes a transition from the first state $\left(\Delta V_{T}>0\right)$ to a second state $\left(\Delta V_{T}<0\right)$. The onset of the second state is prevented by the anharmonic and strongly repulsive forces opposing the perpendicular deformation. The variation of the average friction force $\bar{F}_{f}$ with $k_{z}$ and $F_{N}$ are important aspects revealed in the present model. The variation of $\bar{F}_{f}$ with $k_{z}$ with constant $F_{N}$ is illustrated in Fig. 5(a). $\bar{F}_{f}$ has minimum at $k_{z} \sim 7.5 \mathrm{eV} / \AA^{2}$, which can be explained as follows. In the range $k_{z}<7.5 \mathrm{eV} / \AA^{2}, \bar{F}_{f}$ increases with decreasing $k_{z}$; the softer the material in the perpendicular direction, the higher the energy lost by friction. On the other hand, for $7.5<k_{z}<20 \mathrm{eV} / \AA^{2}$ the energy lost due to friction decreases with decreasing $k_{z} \cdot \bar{F}_{f}$ becomes independent of $k_{z}$, for very large $k_{z}$, where the substrate becomes rigid in the perpendicular direction.
We finally consider the most fundamental aspect i.e., the variation of $\bar{F}_{f}$ with $F_{N}$. This is usually taken as $\bar{F}_{f}=\mu_{k} F_{N}$, which is true for the period of sticking. In the range covering several stick-slip periods the dynamic friction constant $\mu_{d}$ may not be constant. The relation between $\bar{F}_{f}$ and $F_{N}$ obtained in our model depending on $k_{z}$ and anharmonicity of $V_{p, i}$ is illustrated in Fig. 5(b). For a surface that is rigid in the perpendicular direction $\left(k_{z} \rightarrow \infty\right) \mu_{d}$ is constant, but varies with $F_{N}$ when $k_{z}$ is finite. $\bar{F}_{f}$ decreases first and then increases with $F_{N}$ for $k_{z} \sim 10 \mathrm{eV} / \AA^{2}$. This behavior is related to the inversion of corrugation explained above in Fig. 4(b). Depending upon the value of $k_{z}$, the minimum of the $\bar{F}_{f}\left(F_{N}\right)$ curve occurs at different $F_{N}$. For large $F_{N}$, the relation becomes linear. At this point we examine the effect of the anharmonic potential of the substrate atoms in the perpendicular direction $V_{p, i}$. The potential including an anharmonic contribution is expressed in terms of a polynomial up to fourth power of the perpendicular displacement $\left(z_{i}-z_{i 0}\right)$. For small displacements around the equilibrium position $V_{p, i} \sim k_{z}\left(z_{i}-z_{i 0}\right)^{2} / 2$. Our calculations in the model described in Fig. 1 show that the anisotropy of the stick-slip motion disappears gradually with an increasing anharmonic contribution in $V_{p, i}$. As a result, the $\bar{F}_{f}\left(F_{N}\right)$ curve becomes linear for small loading force $F_{N}$. Nevertheless, the nonlinearity appears for large normal loading forces.

\section{CONCLUSION}

In this paper we investigated dry sliding friction by carrying out calculations of stick-slip motion of a single atom on an infinite atomic chain. The most interesting finding of this study is that owing to the elastic deformation, the interaction energy and the force variations are modified. In particular, for certain circumstances the corrugation of the interaction energy can be inverted. We showed that such a situation may give rise to a second state in the stick-slip motion and anisotropy in the hysteresis curve.
${ }^{1}$ F. P. Bowden and D. Tabor, Friction and Lubrication (Methuen, London, 1965).

${ }^{2}$ E. Rabinowicz, Friction and Wear (Wiley, New York, 1965).

${ }^{3}$ J. N. Israelachvili, P. M. McGuiggan, and H. M. Homola, Science 240, 189 (1987).

${ }^{4}$ For current references see, for example, M. Grunze and H. J. Kreuzer, in Adhesion and Friction, edited by Editor(s), Springer Series in Surface Sciences Vol. 17 (Springer-Verlag, Berlin, 1989); B. Bhushan, J. N. Israelachvili, and U. Landman, Nature 347, 607 (1995).

${ }^{5}$ G. Binnig, C. F. Quate, and Ch. Gerber, Phys. Rev. Lett. 56, 930 (1986); G. Binnig, Ch. Gerber, E. Stoll, T. R. Albrecht, and C. F. Quate, Europhys. Lett. 3, 1281 (1987).

${ }^{6}$ C. M. Mate, G. M. McClelland, R. Erlandsson, and S. Chiang, Phys. Rev. Lett. 59, 1942 (1987).

${ }^{7}$ E. Meyer, R. Overney, D. Brodbeck, L. Howald, R. Lüth, J. Frommer, and H. J. Güntherodt, Phys. Rev. Lett. 69, 1777 (1992)
${ }^{8}$ Forces in Scanning Probe Methods, Vol. 286 of NATO Advanced Study Institute, Series E: Applied Science, edited by H. J. Güntherodt, D. Anselmetti, and E. Meyer (Kluwer, Dordrecht, 1995).

${ }^{9}$ O. Marti, Surf. Coat. Technol. 62, 510 (1993).

${ }^{10}$ R. M. Overney, H. Takano, M. Fujihira, W. Paulus, and H. Ringsdorf, Phys. Rev. Lett. 72, 3546 (1994).

${ }^{11}$ For recent references, see Physics of Sliding Friction, Vol. 311 of NATO Advanced Study Institute, Series E: Applied Science, edited by B. N. J. Persson and E. Tosatti (Kluwer, Dordrecht, 1996).

${ }^{12}$ H. Matsukawa and H. Fukuyama, Phys. Rev. B 49, 17286 (1994).

${ }^{13}$ G. A. Tomlinson, Philos. Mag. 7, 905 (1929).

${ }^{14}$ J. Frenkel and T. Kontorova, Phys. Z. Sowjet. 13, 1 (1938); W. Atkinson and N. Cabrera, Phys. Rev. 138, A763 (1965).

${ }^{15}$ J. B. Sokoloff, Thin Solid Films 206, 208 (1991); Phys. Rev. B 47, 6106 (1993). 
${ }^{16}$ D. Tomanek, W. Zhong, and H. Thomas, Europhys. Lett. 15, 887 (1991).

${ }^{17}$ B. N. J. Persson, Phys. Rev. Lett. 71, 1212 (1993); Phys. Rev. B 50, 4771 (1994).

${ }^{18}$ T. Gyalog, M. Bammerlin, R. Lüthi, E. Meyer, and H. Thomas, Europhys. Lett. 31, 269 (1995).

${ }^{19}$ U. Landman, W. D. Luedtke, and A. Nitzan, Surf. Sci. 210, L177 (1989); U. Landman, W. D. Luedtke, N. A. Burnhan, and R. J. Colton, Science 248, 454 (1990).

${ }^{20}$ P. A. Thomson and M. O. Robbins, Phys. Rev. A 41, 6830 (1990); Science 250, 792 (1990).

${ }^{21}$ J. A. Nieminen, A. P. Sutton, and J. B. Pethica, Acta Metall.
Mater. 40, 2503 (1992); J. A. Nieminen, A. P. Sutton, J. B. Pethica, and K. Kaski, Model. Simul. Mater. Sci. Eng. 1, 83 (1992).

${ }^{22}$ H. Yoshizawa, P. McGuiggan, and J. N. Israelachvili, Science 259, 1305 (1993).

${ }^{23}$ J. B. Sokoloff, Phys. Rev. Lett. 71, 3450 (1993).

${ }^{24} \mathrm{~A}$ detailed analysis of the short-range interactions based on $a b$ initio calculations can be found in S. Ciraci, A. Baratoff, and I. P. Batra, Phys. Rev. B 41, 2763 (1990); S. Ciraci, in Basic Concepts and Applications of Scanning Tunneling Microscopy and Related Techniques, edited by J. Behm, H. Rohrer, and N. Garcia (Kluwer, Dordrecht, 1990), p. 113. 